\title{
High-resolution simulations of particle-driven gravity currents
}

\author{
F. Necker ${ }^{\text {a }}$, C. Härtel ${ }^{\mathrm{a}, *}$, L. Kleiser ${ }^{\text {a }}$, E. Meiburg ${ }^{\mathrm{b}}$ \\ ${ }^{a}$ ETH, Institute of Fluid Dynamics, ETH Zentrum, CH-8092 Zürich, Switzerland \\ ${ }^{\mathrm{b}}$ Department of Mechanical and Environmental Engineering, UCSB, Santa Barbara, CA 93106, USA
}

Received 14 November 2000; received in revised form 29 August 2001

\begin{abstract}
High-resolution simulations are presented of particle-driven gravity currents in the lock-exchange configuration. The study concentrates on dilute flows with small density differences between particle-laden and clear fluid. Moreover, particles are considered which have negligible inertia, and which are much smaller than the smallest length scales of the buoyancy-induced fluid motion. For the mathematical description of the particulate phase a Eulerian approach is employed with a transport equation for the local particlenumber density. The governing equations are integrated numerically with a high-order mixed spectral/ spectral-element technique. In the analysis of the results, special emphasis is placed on the sedimentation of particles and the influence of particle settling on the flow dynamics. Time-dependent sedimentation profiles at the channel floor are presented which agree closely with available experimental data. A detailed study is conducted of the balance between the various components of the energy budget of the flow, i.e. the potential and kinetic energy, and the dissipative losses. Furthermore, the simulation results, along with a modified Shields criterion, are used to show that resuspension of sediment back into the particle-driven current is unlikely to occur in the cases considered. Two-dimensional (2D) and three-dimensional (3D) computations are compared which reveal that, for the present configuration, a 2D model can predict reliably the flow development at early times. However, concerning the long-time evolution of the flow, more substantial differences exist between 2D and 3D simulations. (C) 2002 Elsevier Science Ltd. All rights reserved.
\end{abstract}

Keywords: Gravity current; Lock-exchange flow; Numerical simulation

\section{Introduction}

Gravity currents, which form when a heavier fluid propagates into a lighter one, are frequently encountered both in the environment as well as in engineering applications (Huppert, 1986;

\footnotetext{
Corresponding author.

E-mail address: carlos.haertel@power.alstom.com (C. Härtel).
} 
Simpson, 1997). Particle-driven gravity currents form a special class of these flows, as their density difference is caused by differential loading with suspended particles. Turbidity currents in oceans or lakes, which can lead to substantial erosion and transport of sediment, are typical examples of such flows in geophysical situations (see Parker et al., 1986; Middleton, 1993; Dade and Huppert, 1994). Another well-known example are powder snow avalanches on sloping terrain which pose a serious threat in mountain areas (Issler et al., 1998). Clearly, the ability to predict the relevant features of such flows, such as the speed or the final runout length, is of great importance in practice.

To study the dynamics of particle-driven gravity currents under controlled conditions, extensive laboratory experiments were conducted. Most of the experiments were concerned with the spreading of particulate suspensions released into a large body of clear fluid in rectangular water tanks (Bonnecaze et al., 1993; de Rooij and Dalziel, 1998; Gladstone et al., 1998). Quantities that were monitored in the experiments include the speed and height of the propagating fronts, or the shape of the sediment layer that forms beneath the current due to particle settling. Knowledge of such global flow characteristics has proven very valuable in the assessment of simplified theoretical approaches, like box models and shallow-water theory, that were developed to better understand the basic phenomena involved (Dade and Huppert, 1995; Hallworth et al., 1998; Hogg et al., 2000). However, detailed measurements of velocity fields or particle concentrations could not be undertaken, and therefore some fundamental aspects of particulate gravity currents are still little understood today. Among these are the mixing within the current, the progressive segregation of particles and carrier fluid due to sedimentation, and the overall balance of potential energy, fluid motion, and molecular dissipation. In this respect, accurate numerical simulations can be helpful in obtaining the detailed insight into the flow which is needed to fill the present gaps in knowledge.

The current paper discusses work in which particle-driven gravity currents are studied by means of high-resolution simulations which account for all relevant time and length scales of the flow. In

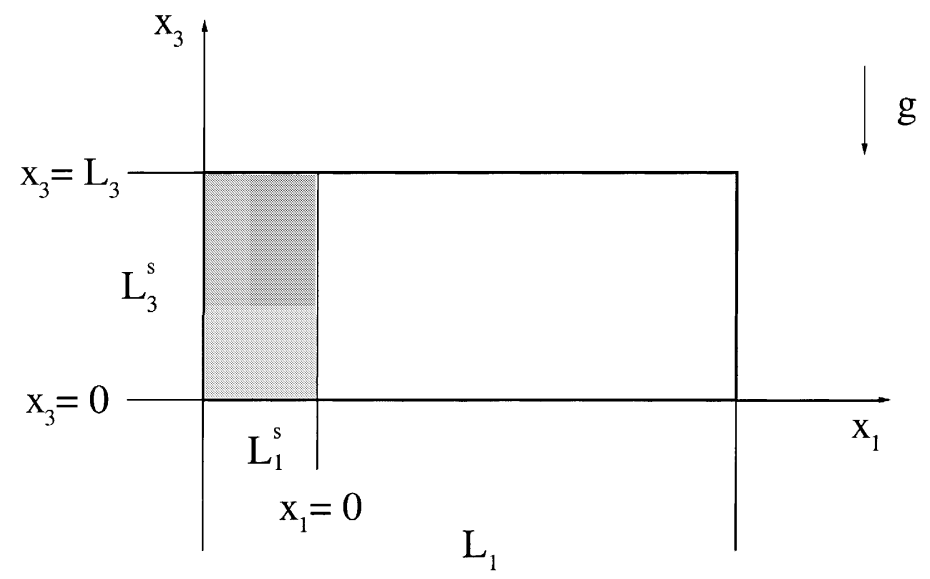

Fig. 1. Sketch of the initial set-up of a lock-exchange flow in a plane channel of length $L_{1}$, width $L_{2}$, and height $L_{3}$ (gravity acts in the vertical direction). Initially a subvolume of the channel (length $L_{1}^{\mathrm{s}}$, height $L_{3}^{\mathrm{s}}=L_{3}$ ) is filled with particle-laden fluid. The remaining part of the channel contains clear fluid which is separated from the suspension by a vertical splitter plate located at $x_{1}=0$. After removing the plate a mutual intrusion flow develops driven by the difference in density between particle-laden and clear fluid. 
the simulations the prototype configuration of a lock-exchange flow is considered, which has been studied in numerous experiments in the past (see Simpson, 1997). The basic set-up consisting of a plane channel filled with suspension and clear fluid at rest is sketched in Fig. 1. In the laboratory, a vertical splitter plate is used to separate the fluids before the start of the experiment. When the plate is withdrawn, a mutual intrusion flow develops in which the particle-laden front travels along the bottom to the right. In the present simulations the particulate phase will be treated in a Eulerian fashion using a transport equation for the local particle-number density. This approach is appropriate for the case of dilute suspensions of small particles on which we want to concentrate here.

Before we discuss the simulation results, we will briefly outline in Section 2 the governing equations. Subsequently, in Section 3 the flow structure of a particle-driven front will be discussed, as it is obtained from a three-dimensional simulation. Section 4 is then concerned with the sedimentation of particles and its influence on the flow dynamics. Among other things, we will analyze the temporal evolution of the rate of particle deposition and of the sediment layer formed beneath the current. Also, the energy losses associated with particle settling will be investigated, in order to quantify how much of the initially available potential energy is eventually converted into convective transport and fine-scale turbulence. Finally, in Section 6 the simulation results will be used, along with a modified Shields criterion (Mantz, 1973; Yalin and Karahan, 1979), to show that resuspension of sediment back into the flow is unlikely to occur in the cases considered. The present analysis employs both two-dimensional (2D) and three-dimensional (3D) simulations, and a direct comparison of $2 \mathrm{D}$ and 3D results will be performed to assess which features of a particledriven gravity current can be captured by a $2 \mathrm{D}$ model.

\section{Governing equations}

We focus on dilute suspensions here with volume fractions well below 1\%. In this case, particleparticle interactions can be neglected and the coupling between particle and fluid motion is dominated by the transfer of momentum, rather than volumetric displacement effects. In consequence, the fluid velocity field $\tilde{u}_{\mathrm{i}}$ (a tilde denotes a dimensional quantity here) can be assumed as divergence free, i.e.

$$
\frac{\partial \tilde{u}_{l}}{\partial \tilde{x}_{l}}=0
$$

In the present investigation we will only consider particles with a diameter $\tilde{d}_{\mathrm{p}}$ which is much smaller than the smallest length scales of the fluid motion induced by the buoyancy forces. Furthermore, the density of the particles $\tilde{\rho}_{\mathrm{p}}$ is assumed to be significantly higher than the density of the fluid $\tilde{\rho}$ such that the dominant flow force on an individual particle is the Stokes $\operatorname{drag} \tilde{F}_{i}$ (for a general discussion of flow forces acting on small particles - and of their relative importance - see e.g. Maxey and Riley, 1983; Lázaro and Lasheras, 1989). For spherical particles with small particle Reynolds numbers the Stokes drag force reads

$$
\tilde{F}_{i}=3 \pi \tilde{\mu} \tilde{d}_{\mathrm{p}}\left(\tilde{u}_{\mathrm{i}}-\tilde{u}_{\mathrm{p}, \mathrm{i}}\right)
$$


where $\tilde{\mu}$ is the dynamic viscosity of the fluid, and $\left(\tilde{u}_{\mathrm{i}}-\tilde{u}_{\mathrm{p}, \mathrm{i}}\right)$ is the difference between the fluid velocity and the velocity of the particle $\tilde{u}_{\mathrm{p}, \mathrm{i}}$. Particles with negligible inertia, that is with an aerodynamic response time significantly smaller than the characteristic time scale of the flow, move with a velocity that is equal to the sum of the local fluid velocity and a settling speed $\tilde{u}_{\mathrm{s}}$, i.e.,

$$
\tilde{u}_{\mathrm{p}, \mathrm{i}}=\tilde{u}_{\mathrm{i}}+\tilde{u}_{\mathrm{s}} e_{\mathrm{i}}^{\mathrm{g}},
$$

where $\underline{e}^{\mathrm{g}}=(0,0,-1)$ is the vector pointing in the direction of gravity (see Fig. 1$)$. The settling velocity is obtained by balancing the gravitational forces with the Stokes drag of a single freefalling spherical particle and reads ( $\tilde{g}$ denotes the gravitational acceleration)

$$
\tilde{u}_{\mathrm{s}}=\frac{\tilde{d}_{\mathrm{p}}^{2}\left(\tilde{\rho}_{\mathrm{p}}-\tilde{\rho}\right) \tilde{g}}{18 \tilde{\mu}} .
$$

The assumption that the particle velocity is equal to the sum of fluid and settling velocities implies that the particle velocity field is also divergence free and, hence, that particles do not accumulate. The particle field can then be treated in a Eulerian manner, and its temporal evolution described by a transport equation

$$
\frac{\partial \tilde{c}}{\partial \tilde{t}}+\left(\tilde{u}_{l}+\tilde{u}_{s} e_{l}^{\mathrm{g}}\right) \frac{\partial \tilde{c}}{\partial \tilde{x}_{l}}=\tilde{\kappa} \frac{\partial^{2} \tilde{c}}{\partial \tilde{x}_{l} \partial \tilde{x}_{l}},
$$

where $\tilde{c}$ is the particle-number density which, for simplicity, will be referred to as the concentration in the remainder. The diffusion term on the right-hand side of Eq. (5), with a diffusivity $\tilde{\kappa}$, has been included to account for the fact that sharp concentration interfaces in particulate suspensions usually spread with time. This spreading may either be caused by hydrodynamic diffusion of particles (see Davis and Hassen, 1988; Ham and Homsy, 1988) or by the polydisperse nature of suspensions which usually contain particles of non-uniform size (and, hence, non-uniform settling speeds). The details of this diffusion-like spreading of the interface depend on the flow situation considered, but we have not attempted here to match $\tilde{\kappa}$ to a particular experiment or application. Rather we set $\tilde{\kappa}=\tilde{v}$, since from simulations of density-driven gravity currents (see Härtel et al., 2000a) we found that diffusion in the concentration field has a negligible influence on the relevant flow quantities, as long as $\tilde{\kappa}$ is not much larger than the kinematic viscosity of the fluid.

To describe the motion of the fluid phase, the incompressible Navier-Stokes equations are employed here which have to be augmented by a forcing term that accounts for two-way coupling, i.e. the effect of the particles on the fluid. Since we are interested in particulate flows with small mass loadings, we make use of the Boussinesq approximation that density variations are important only when multiplied by gravitational terms (cf. Bonnecaze et al., 1993; Hogg et al., 2000). The governing equations then read in dimensional form

$$
\frac{\partial \tilde{u}_{\mathrm{i}}}{\partial \tilde{t}}+\tilde{u}_{l} \frac{\partial \tilde{u}_{\mathrm{i}}}{\partial \tilde{x}_{l}}=-\frac{1}{\tilde{\rho}} \frac{\partial \tilde{p}}{\partial \tilde{x}_{\mathrm{i}}}+\tilde{v} \frac{\partial^{2} \tilde{u}_{\mathrm{i}}}{\partial \tilde{x}_{l} \partial \tilde{x}_{l}}-\frac{\tilde{c}}{\tilde{\rho}} \tilde{F}_{i},
$$

where $\tilde{p}$ denotes the pressure and $\tilde{v}$ is the kinematic viscosity.

In rendering the above equations dimensionless, we choose $\tilde{L}_{3} / 2$ as a characteristic length scale. This choice is motivated by the fact that, during the initial phase of the flow, the height of the 
front is approximately equal to half the channel height. As the characteristic velocity scale we employ the buoyancy velocity $\tilde{u}_{\mathrm{b}}$ which is defined as

$$
\tilde{u}_{\mathrm{b}}=\sqrt{\tilde{g}^{\prime} \tilde{L}_{3} / 2},
$$

where $\tilde{g}^{\prime}$ is the reduced gravitational acceleration

$$
\tilde{g}^{\prime}=\frac{\pi\left(\tilde{\rho}_{\mathrm{p}}-\tilde{\rho}\right) \tilde{c}_{0} \tilde{d}_{\mathrm{p}}^{3}}{6 \tilde{\rho}} \tilde{g},
$$

and $\tilde{c}_{0}$ is the initial (homogeneous) concentration of the suspension. The dimensionless equations then read

$$
\begin{aligned}
& \frac{\partial u_{l}}{\partial x_{l}}=0, \\
& \frac{\partial u_{\mathrm{i}}}{\partial t}+u_{l} \frac{\partial u_{\mathrm{i}}}{\partial x_{l}}=-\frac{\partial p}{\partial x_{\mathrm{i}}}+\frac{1}{\sqrt{G r}} \frac{\partial^{2} u_{\mathrm{i}}}{\partial x_{l} \partial x_{l}}+c e_{\mathrm{i}}^{\mathrm{g}}, \\
& \frac{\partial c}{\partial t}+\left(u_{l}+u_{\mathrm{s}} e_{l}^{\mathrm{g}}\right) \frac{\partial c}{\partial x_{l}}=\frac{1}{\sqrt{S c^{2} G r}} \frac{\partial^{2} c}{\partial x_{l} \partial x_{l}} .
\end{aligned}
$$

In Eqs. (10) and (11), $c=\tilde{c} / \tilde{c}_{0}$, and the dimensionless pressure is given by $p=\tilde{p} / \tilde{\rho} \tilde{u}_{\mathrm{b}}^{2}$. Three dimensionless parameters appear in the above equations, namely the Grashof number $G r$, the Schmidt number $S c$, and the dimensionless settling velocity of the particles $u_{\mathrm{s}}$, which are defined as

$$
\begin{aligned}
& G r=\left(\frac{\tilde{u}_{\mathrm{b}} \tilde{L}_{3} / 2}{\tilde{v}}\right)^{2}, \\
& S c=\frac{\tilde{v}}{\tilde{\kappa}}, \\
& u_{\mathrm{s}}=\frac{\tilde{u}_{\mathrm{s}}}{\tilde{u}_{\mathrm{b}}} .
\end{aligned}
$$

The Grashof number represents the ratio of buoyancy forces and viscous forces in the fluid. The Schmidt number compares the diffusivities in the velocity and the density fields, and is generally set to unity here. The dimensionless velocity $u_{\mathrm{s}}$ quantifies the relative importance of particle settling. The larger the values of $u_{\mathrm{s}}$, the earlier the flow will deviate from a density-driven gravity current (that corresponds to the case $u_{\mathrm{s}}=0$ ). In the present work, the settling speed is set to $u_{\mathrm{s}}=0.02$ in order to match the conditions in the experiments of de Rooij and Dalziel (1998) that we will use for comparison with our numerical results in Section 4.

To integrate the governing equations in the flow domain sketched in Fig. 1, we employ a numerical scheme based on spectral/spectral-element discretizations in space along with finite differences in time. A detailed description of the computational approach and its validation is given in Härtel et al. (1997, 2000a). Due to the choice of a Fourier spectral scheme in $x_{1}$ and $x_{2}$, the lateral boundaries of the flow domain are treated as symmetry planes in both the velocity and the concentration field. At top and bottom of the computational domain, no-slip walls are prescribed in the simulations, that is $u_{\mathrm{i}}=0$. We remark that at first sight the use of free-slip conditions at the 
top boundary may appear more natural, given the fact that in corresponding experiments open channels are used. However, in laboratory flows impurities usually make a free surface act as a no-slip surface (see Britter and Simpson, 1978). For the concentration field, we use a no-flux boundary condition at the top boundary, which ensures that no particulate matter is transported across this plane. This is achieved by imposing

$$
u_{\mathrm{s}} c-\frac{1}{\sqrt{S c^{2} G r}} \frac{\partial c}{\partial x_{3}}=0 \quad \text { at } x_{3}=2 .
$$

Concerning the bottom of the channel, we assume that the particles leave the flow due to sedimentation. Numerically this is accomplished by an 'outflow' boundary condition, i.e. a convective

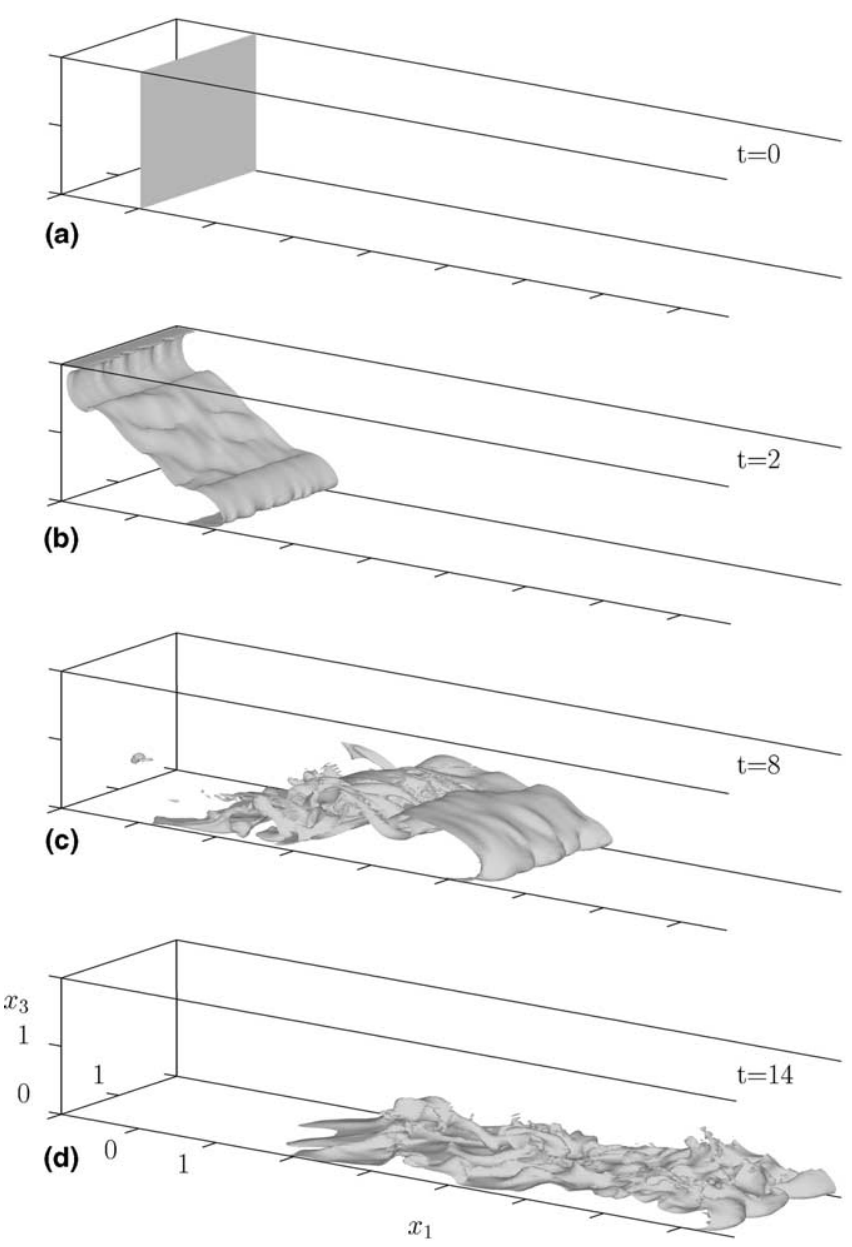

Fig. 2. Structure of a particle-driven gravity current visualized by isosurfaces of concentration at $t=0$ (a), $t=2$ (b), $t=8$ (c), and $t=14$ (d). Results obtained from a 3D simulation for a Grashof number of $G r=5 \times 10^{6}$ and a dimensionless settling velocity of $u_{\mathrm{s}}=0.02$. In all cases, an isovalue of 0.25 is employed. Note that at $t=14$ the concentration in the rear part of the channel has almost dropped to zero. 
boundary condition with the settling speed taken as the characteristic velocity. This is, we set at the lower boundary

$$
\frac{\partial c}{\partial t}=u_{\mathrm{s}} \frac{\partial c}{\partial x_{3}} \quad \text { at } x_{3}=0
$$

Clearly, the applicability of this approach is confined to situations where no resuspension of particles back into the flow occurs, a point that we will return to in Section 6.

\section{Structure of the front}

To illustrate the spatial structure and the temporal evolution of a particle-driven front, Fig. 2 gives isosurfaces of constant particle concentration as obtained from a 3D simulation. The Grashof number of the flow is $G r=5 \times 10^{6}$, and the lateral size of the flow domain was set to $L_{2}=2$. For the length $L_{1}$ of the flow domain we have chosen $L_{1}=18$ which is slightly more than the final runout length of the front. For the numerical discretization a mesh consisting of a maximum number of $N_{1} \times N_{2} \times N_{3}=1440 \times 200 \times 221$ grid points was used. As initial condition, fluid at rest was prescribed with the suspension part being confined to a subvolume of aspect ratio $L_{1}^{\mathrm{s}} / L_{3}^{\mathrm{s}}=1 / 2$ (see Fig. 1). Unless stated otherwise, this aspect ratio is used in all simulations presented in the current study. In order to enhance the breakdown of the flow into a 3D state after the release, weak turbulent disturbances in the velocity field are superimposed at time $t=0$ in the neighborhood of the interface. This turbulence field was obtained from a separate simulation of decaying turbulence that was conducted in a very short computational domain of length $L_{1}=0.2$. An impression of the initial turbulence can be gained from Fig. 3(a) where isosurfaces of the
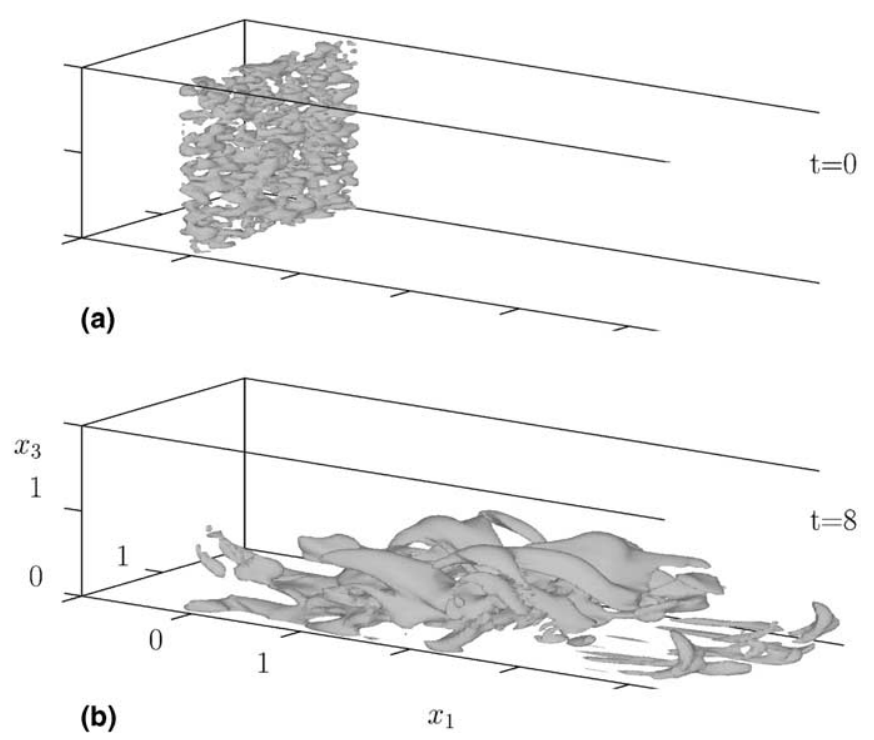

Fig. 3. Isosurfaces of spanwise velocity at two different times $t$ of the simulation (same flow as in Fig. 2). (a) $t=0$, isovalue set to 0.02 . (b) $t=8$, isovalue set to 0.06 (corresponding to about $10 \%$ of the front velocity at this time). 
spanwise velocity component are depicted. The kinetic energy contained in the turbulence field is very small and amounts to merely $0.5 \%$ of the potential energy in the flow domain at $t=0$.

For a short time interval after the release, the flow is dominated by the development of a pair of start-up vortices, a phenomenon which was studied in detail by Härtel et al. (1999). These start-up vortices are responsible for the sharp kink in the isosurface of concentration at time $t=2$ which separates the developing heads of the fronts from the almost planar interface in the channel interior. During the further evolution of the flow, the light front in the upper channel half encounters the left boundary and is reflected, thereby leading to a strong downward motion that drastically reduces the thickness of the suspension layer in the rear part of the channel. At time $t=8$, when the particulate front is fully developed, its head features the typical lobe-and-cleft instability shown in a close-up in Fig. 4. The lobe-and-cleft structure goes along with a strong three-dimensionality in the flow. This is seen in Fig. 3(b) where the isosurface of $u_{2}$ at $t=8$ reveals that intense spanwise motions exist not only in the wake of the head, but also at the foremost part. At this early stage of the flow development, the structure at the leading edge is still very similar to what is seen in the density-driven case (see Simpson, 1972; Härtel et al., 2000a,b). However, after about $t=10$, the current becomes increasingly affected by the settling of particles which, among other things, makes the pronounced head of the front gradually disappear (see Fig. 2(d)).

To illustrate more clearly how a particle-driven front progressively deviates from a densitydriven front, Fig. 5 gives the respective results for the front position $x_{\mathrm{f}}$ as a function of time (for the same initial geometry and density ratio). Until $t \approx 10$, both fronts travel at the same constant speed, but the particle-driven front experiences a significant deceleration at later times. For density-driven gravity currents it is known that the flow initially passes through a slumping phase in which the ratio of front height to channel height plays an important role for the dynamics. This phase is usually followed by a self-similar phase, where the front speed decays with time as $t^{-1 / 3}$ (see Huppert and Simpson, 1980; Rottman and Simpson, 1983). For the density-driven flow in Fig. 5 this slumping phase lasts about 15 dimensionless time units until the front has travelled some 8-10 lock lengths. The particle-driven flow, on the other hand, becomes affected by particle sedimentation already before the end of the slumping phase, meaning that a self-similar regime

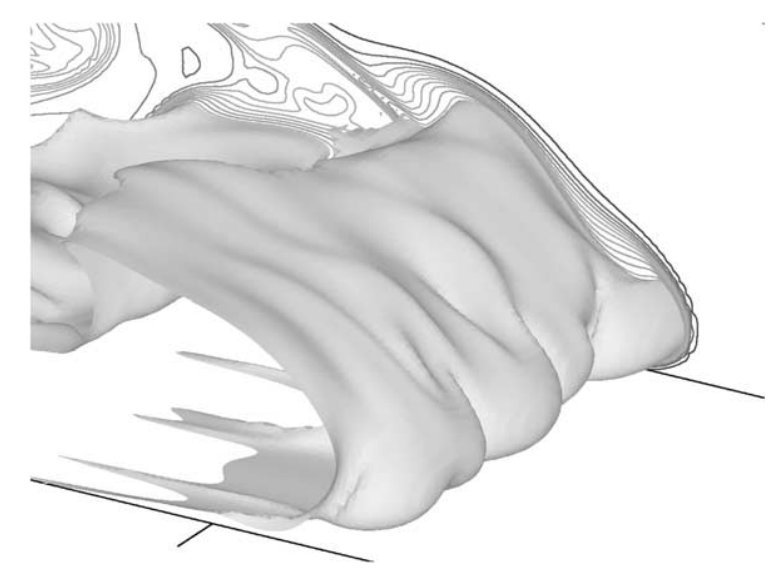

Fig. 4. Close-up of the nose region of the particulate front in Fig. 2(c). Visualization by an isosurface of concentration (isovalue: 0.25 ) and isolines of concentration in the back plane. 


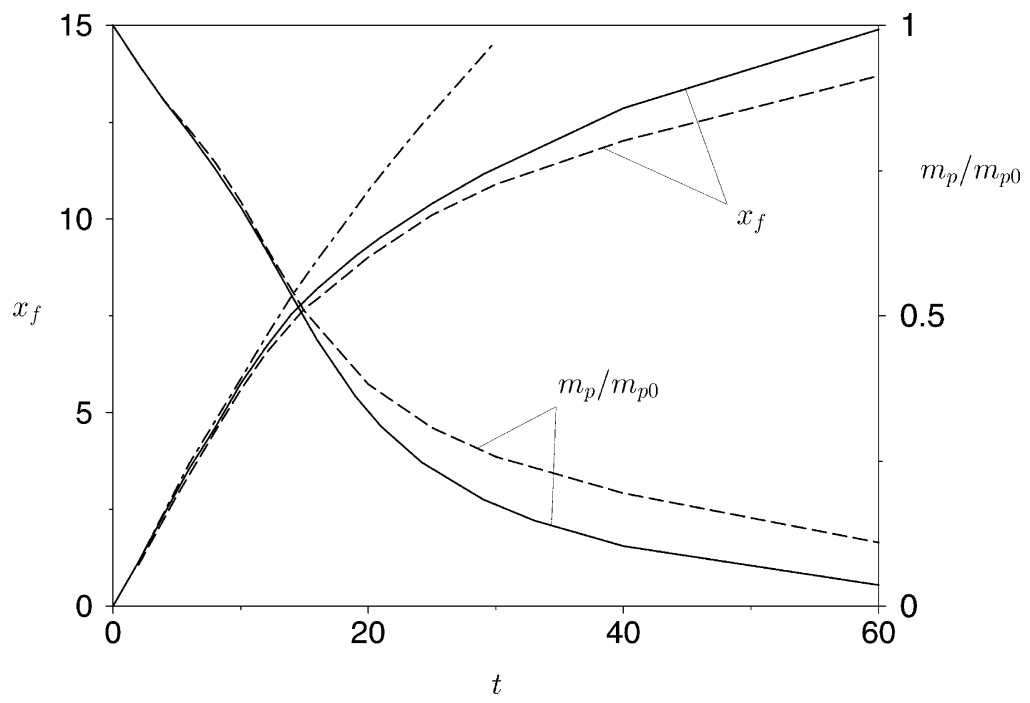

Fig. 5. Front position $x_{\mathrm{f}}$ as a function of time, and temporal evolution of the mass $m_{\mathrm{p}}$ of suspended particles normalized with the initial mass $m_{\mathrm{p} 0}$. Comparison of results obtained from 3D simulation (solid lines, same flow as in Fig. 2 ) and 2D simulation (dashed lines). The dot-dashed line gives the front position $x_{\mathrm{f}}(t)$ as obtained from a 3D simulation of a density-driven gravity current $\left(u_{\mathrm{s}}=0.0\right)$ with the same Grashof number as the particle-driven flow.

does not develop. Rather, the front speed quickly decays after $t \approx 10$, and the front eventually comes to rest having a final runout length of about 16 units. From the time history of the mass $m_{\mathrm{p}}$ of suspended particles in the flow, which is included in Fig. 5, it seen that the influence of sedimentation on the spreading of the front becomes discernible after about half of the particles have settled.

During the early stages, the flow exhibits a strong spanwise coherence due to the start-up vortices and the billows which are shed from the advancing fronts. However, already at $t=8$ this coherence in the flow has largely disappeared and a turbulent state with a broad range of excited scales is attained. This is demonstrated in Fig. 6, where the spanwise spectra $E_{\mathrm{i}}$ of the three velocity components $u_{\mathrm{i}}$ are displayed together with the spectrum $E_{\mathrm{c}}$ of the concentration field. These are defined as

$$
\begin{aligned}
& E_{\mathrm{i}}\left(k_{2}\right)=\int_{0}^{L_{1}} \int_{0}^{L_{3}}\left|\hat{u}_{\mathrm{i}}\left(x_{1}, k_{2}, x_{3}\right)\right|^{2} \mathrm{~d} x_{3} \mathrm{~d} x_{1}, \\
& E_{\mathrm{c}}\left(k_{2}\right)=\int_{0}^{L_{1}} \int_{0}^{L_{3}}\left|\hat{c}\left(x_{1}, k_{2}, x_{3}\right)\right|^{2} \mathrm{~d} x_{3} \mathrm{~d} x_{1},
\end{aligned}
$$

where, respectively, $\hat{u}_{\mathrm{i}}$ and $\hat{c}$ are the coefficients obtained from a spanwise Fourier transformation of the velocity and density fields, i.e. $\left(\mathrm{I}=\sqrt{-1}, \alpha_{2}=\pi / L_{2}\right)$

$$
f=\sum \hat{f}_{k} \exp \left(\mathrm{I} \alpha_{2} k_{2} x_{2}\right), \quad f=u_{\mathrm{i}}, c .
$$

Besides illustrating the broad-banded turbulent spectrum, Fig. 6 also shows that in the simulation the flow field is fully resolved, the decay of the spectra with increasing $k_{2}$ covering seven to eight 


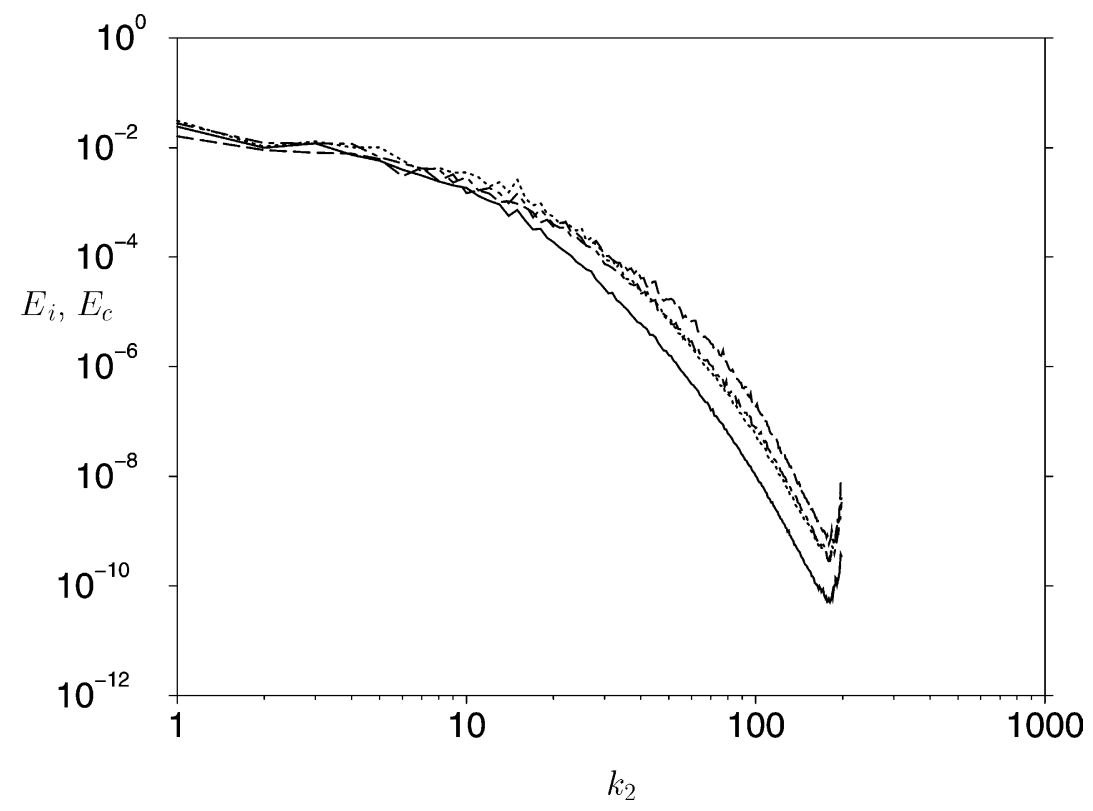

Fig. 6. Spanwise modal energy according to Eqs. (17) and (18) as a function of $k_{2}: E_{1}$ (dot-dashed), $E_{2}$ (solid), $E_{3}$ (dotted), $E_{\mathrm{c}}$ (dashed). Results for the flow shown in Fig. 2 at time $t=8$.

decades. At later times of the flow, when the turbulent energy slowly decreases, the numerical resolution requirements also decrease, which allows using coarser meshes. In fact, the finest mesh is required in the initial adjustment phase of the flow that lasts until about $t=10$.

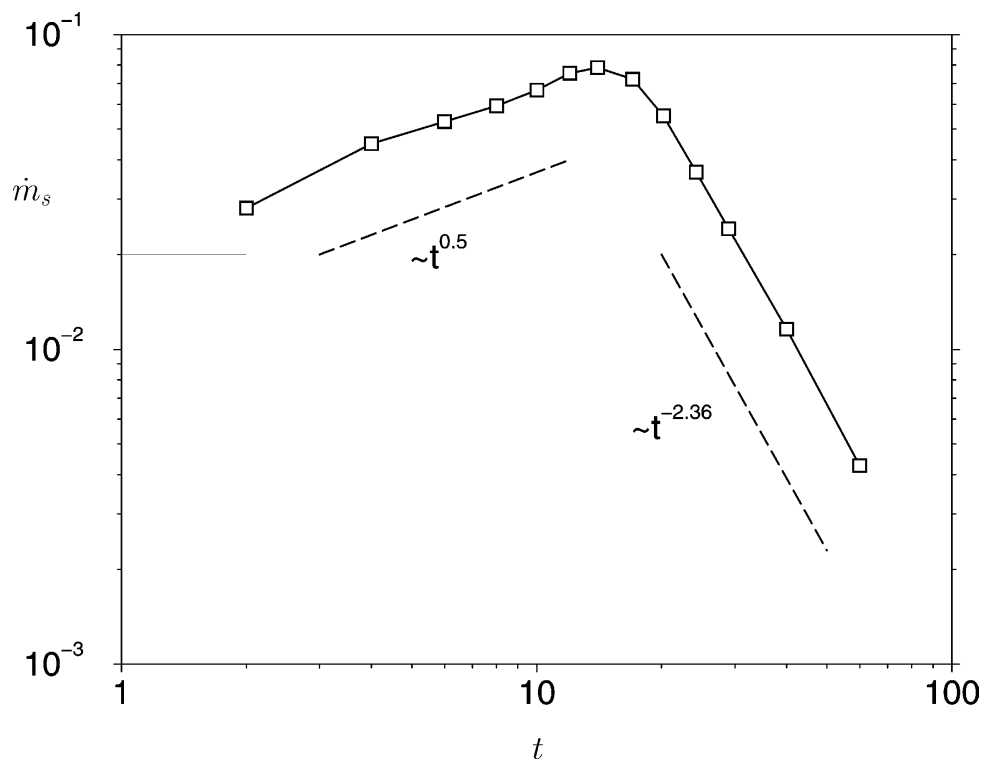

Fig. 7. Sedimentation rate $\dot{m}_{\mathrm{s}}(t)$ (see Eq. (20)) at bottom wall of the channel as a function of time. Same flow as in Fig. 2. 


\section{Sedimentation}

The settling of particles leads to a continued loss of suspended material in the flow. In consequence, at the bottom of the tank a sediment layer forms that grows with time. The total amount of particles lost can be inferred from the curve in Fig. 5 which gives the time history of the mass of particles suspended in the fluid. It is seen that the sedimentation process is very rapid during the first 10-20 dimensionless time units until about $70 \%$ of all particles have settled out. After that, the sedimentation slows down substantially. Fig. 7 gives the time history of the sedimentation rate $\dot{m}_{\mathrm{s}}$, which is defined here as the time derivative of the total mass $m_{\mathrm{s}}$ of sedimented particles per unit span

$$
\dot{m}_{\mathrm{s}}(t)=\frac{\mathrm{d} m_{\mathrm{s}}(t)}{\mathrm{d} t}=\frac{1}{L_{2}} \int_{0}^{L_{1}} \int_{0}^{L_{2}} c_{w}\left(x_{1}, x_{2}, t\right) u_{\mathrm{s}} \mathrm{d} x_{2} \mathrm{~d} x_{1},
$$

where $c_{w}=c\left(x_{3}=0\right)$ is the concentration at the bottom wall. The initial value of the sedimentation rate $\dot{m}_{\mathrm{s}}(t=0)=0.02$ is indicated in the graph by a thin horizontal line. Up to about $t=14$, the sedimentation rate steadily increases, reflecting the fact that the suspension stretches out along the bottom while remaining almost undiluted. In the logarithmic representation this increase is seen to be roughly proportional to $t^{0.5}$. A striking feature of the sedimentation rate is the abrupt change that follows the steady increase leading to a massive decay of $\dot{m}_{\mathrm{s}}$ with time. This change occurs when about half the particles have settled at the bottom, and it coincides with the time when the front speed of the particle-driven current starts to deviate from the speed of its densitydriven counterpart. In the subsequent flow development, the time history of the sedimentation rate again approaches a power law. However, this time a rather large negative exponent is found with an absolute value between 2.3 and 2.4.

For the sake of a direct comparison of computational results with experimental data, we have evaluated the integrated sedimentation profile at the bottom for selected times from a $2 \mathrm{D}$ simulation at $G r=10^{8}$. For this Grashof number, measurements of de Rooij and Dalziel (1998) are available. The small, heavy particles used in the experiments have a non-dimensional settling speed of about 0.02 , which is also used in the simulation. We remark here that a $3 \mathrm{D}$ simulation at such high Grashof number is infeasible at present due to the excessive resolution requirements. However, as will be shown in Section 5, differences between a 2D and a 3D simulation are small as far as the spatial structure of the sediment layer is concerned. In Fig. 8 the computational and experimental sedimentation profiles for three different time instants are given in the form of a nondimensional mean deposit $D_{t}\left(x_{1}, t\right)$ per unit span. The curves are normalized such that the results for the latest time $(t \rightarrow \infty)$, when all particles have already settled out, integrate to unity. For the computational data this is equivalent to

$$
D_{t}\left(x_{1}, t\right)=\frac{1}{L_{1}^{\mathrm{s}} L_{3}^{\mathrm{s}}} \int_{0}^{t} c_{w}\left(x_{1}, \tau\right) u_{\mathrm{s}} \mathrm{d} \tau
$$

The good agreement between the experimental data and the simulation results, which are represented by dashed and solid lines, respectively, is readily seen. Both consistently show the maximum of the final deposition curve to be located some distance downstream of the initial interface location, a feature that was already observed in the earlier experiments of Bonnecaze 


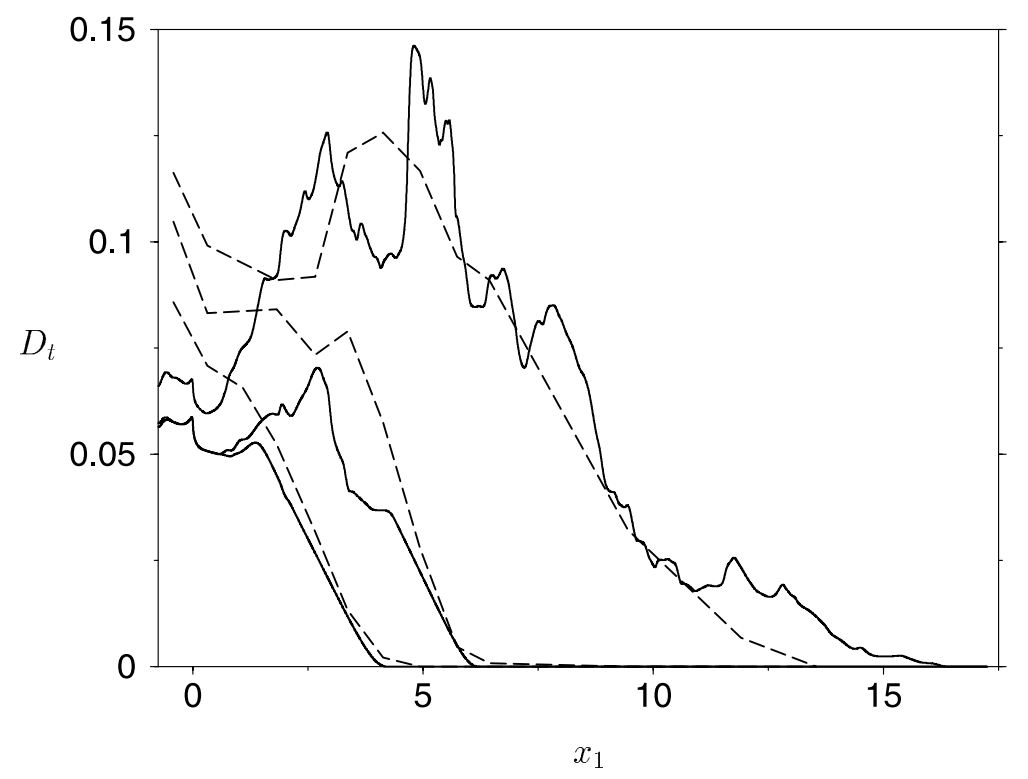

Fig. 8. Non-dimensional particle deposit $D_{t}$ according to Eq. (21) as a function of $x_{1}$. Results for times $t=7.3$, $t=10.95$, and final profile $(t \rightarrow \infty)$ after all particles have settled (all curves normalized such that the curves for the final profile integrate to unity). Solid line: 2D simulation, dashed line: experimental data of de Rooij and Dalziel (1998). In both cases $G r=1 \times 10^{8}, u_{\mathrm{s}}=0.02, L_{1}^{\mathrm{s}}=0.75, L_{3}^{\mathrm{s}}=2$.

et al. (1993). Differences between experiments and simulations are primarily seen in the region around the initial lock. However, in the experiments this region is presumably affected not only by disturbances induced by the initial stirring of the suspension but also by the onset of sedimentation before the start of the experiment.

Of fundamental importance for gravity currents is the conversion of potential energy into fluid motion. The potential energy available is given by the elevation of the center of mass of the denser fluid relative to the light fluid. Gravitational acceleration then initiates a flow that goes along with an increase of kinetic and a decrease of potential energy. In finite-volume releases, where there is no replenishment of potential energy by a source, the fluid motion will ultimately decay as a consequence of viscous dissipation. In this case, the total amount of energy dissipated approaches the initially available potential energy after sufficiently long times. In particle-driven gravity currents the situation is complicated by the fact that dissipation is caused not only by the gradients of the (macroscopic) convective motion, but also by the (microscopic) Stokes flow around each individual particle. The latter dissipation equals the loss of potential energy that particles experience as a consequence of progressive settling. Although in the present simulations this microscopic motion is not resolved, the associated dissipation can be computed from the product of the local concentration and the constant settling speed $u_{\mathrm{s}}$. Denoting this dissipation component by $\varepsilon_{\mathrm{s}}$, the global energy budget of a particle-driven gravity current, as derived from Eqs. (10) and (11), reads

$$
\frac{\mathrm{D}}{\mathrm{D} t}\left(k+E_{\mathrm{p}}\right)=-\varepsilon-\varepsilon_{\mathrm{s}}
$$


where $k$ is the total kinetic energy in the flow and $E_{\mathrm{p}}$ is the total potential energy. $\varepsilon$ denotes the viscous dissipation due to macroscopic fluid motion. The four terms in Eq. (22) are computed as

$$
\begin{aligned}
& k(t)=\int_{\Omega} \frac{1}{2} u_{l} u_{l} \mathrm{~d} V, \\
& E_{\mathrm{p}}(t)=\int_{\Omega} c \cdot x_{3} \mathrm{~d} V, \\
& \varepsilon(t)=\int_{\Omega} \frac{2}{\sqrt{G r}} s_{l m} s_{l m} \mathrm{~d} V, \\
& \varepsilon_{\mathrm{s}}(t)=\int_{\Omega} u_{\mathrm{s}} \cdot c \mathrm{~d} V .
\end{aligned}
$$

In Eqs. (23)-(26), integration is performed over the entire flow domain $\Omega$, and $s_{i j}$ is the rateof-strain tensor of the macroscopic velocity field. If we denote the time integral of the dissipation terms by $E_{\mathrm{d}}$ and $E_{\mathrm{s}}$, respectively,

$$
\begin{aligned}
& E_{\mathrm{S}}(t)=\int_{0}^{t} \varepsilon_{\mathrm{S}}(\tau) \mathrm{d} \tau, \\
& E_{\mathrm{d}}(t)=\int_{0}^{t} \varepsilon(\tau) \mathrm{d} \tau,
\end{aligned}
$$

the integral of Eq. (22), which is the total energy balance of the fluid, reads

$$
E_{\mathrm{p}}+k+E_{\mathrm{s}}+E_{\mathrm{d}}=\text { const. }=E_{\mathrm{p} 0}+k_{0}
$$

with $E_{\mathrm{p} 0}$ and $k_{0}$ denoting the potential and kinetic energies at time $t=0$. Note that in the above derivation we have neglected the effect that diffusion in the density field may have on the potential energy in the system (see e.g. Winters et al., 1995). However, we checked the magnitude of the respective terms from the simulation results and found them to be negligibly small.

The time history of the four terms on the left-hand side of Eq. (29) is shown in Fig. 9 for the 3D calculation (solid lines). It is readily seen from the curves that the first 2-3 time units are dominated by a rapid conversion of potential energy into convective motion which makes $E_{\mathrm{p}}$ drop by almost $70 \%$. However, although the potential energy continues to decrease during the further evolution of the flow, the kinetic energy, after having reached a maximum at $t \approx 5$, also starts to decay due to the growing influence of dissipation. While during the first time units dissipative losses are dominated by particle settling, the macroscopic dissipation $E_{\mathrm{d}}$ overtakes $E_{\mathrm{s}}$ at the time when the front reaches a developed turbulent state. It is seen from the curves that this goes along with a strong increase of the dissipation rate $\varepsilon$ by about a factor of two. At times much later than those shown in the figure, both $k$ and $E_{\mathrm{p}}$ tend to zero; on the other hand, $E_{\mathrm{d}}$ and $E_{\mathrm{s}}$ approach final values that indicate, respectively, how much of the initially available potential energy has been converted into macroscopic flow and how much is dissipated due to settling. For the present case, it turns out that sedimentation causes a 'loss' of more than $40 \%$ of $E_{\mathrm{p} 0}$ which, hence, is not available for convective transport and turbulent mixing. This must be contrasted with a densitydriven flow where $E_{\mathrm{p} 0}$ completely converts into fluid motion during the flow. 

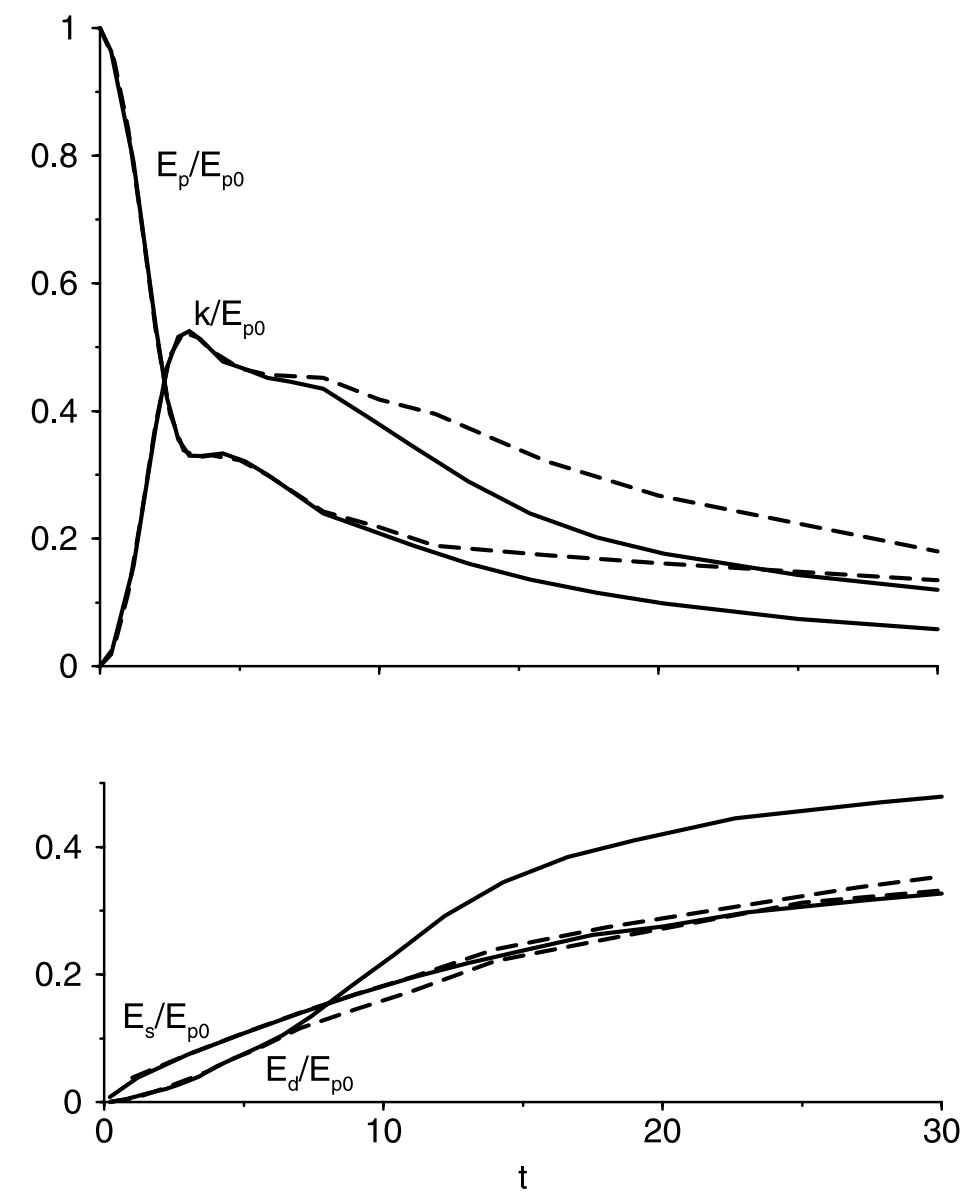

Fig. 9. Time history of kinetic energy $k$, potential energy $E_{\mathrm{p}}$, and the dissipation components $E_{\mathrm{s}}$ and $E_{\mathrm{d}}$. Results normalized with the initial potential energy $E_{p 0}$. Solid lines: 3D simulation (same flow as in Fig. 2), dashed lines: 2D simulation.

\section{Assessment of 2D simulations}

To highlight the potential - and limitations - of 2D simulation models for the study of particulate gravity currents, we will conduct a direct comparison of results obtained from 2D and 3D simulations. In general, 2D simulations are appealing because the number of grid points required is much smaller than for 3D simulations at a given Grashof number. This allows reaching much higher Grashof numbers up to, say, $G r \approx 10^{9}$ at present. However, since a 2D model will miss important flow features, such as the break-up of the interfacial vortices into turbulence, the relevance of findings derived from a 2D simulation is not fully clear in advance. Fig. 10 compares isocontours of concentration for the $3 \mathrm{D}$ calculation discussed before and a $2 \mathrm{D}$ simulation at the same Grashof number. Note that for the 3D case, the spanwise-averaged concentration field is given. Concerning the early flow evolution, $2 \mathrm{D}$ and $3 \mathrm{D}$ simulations produce a very similar mean 

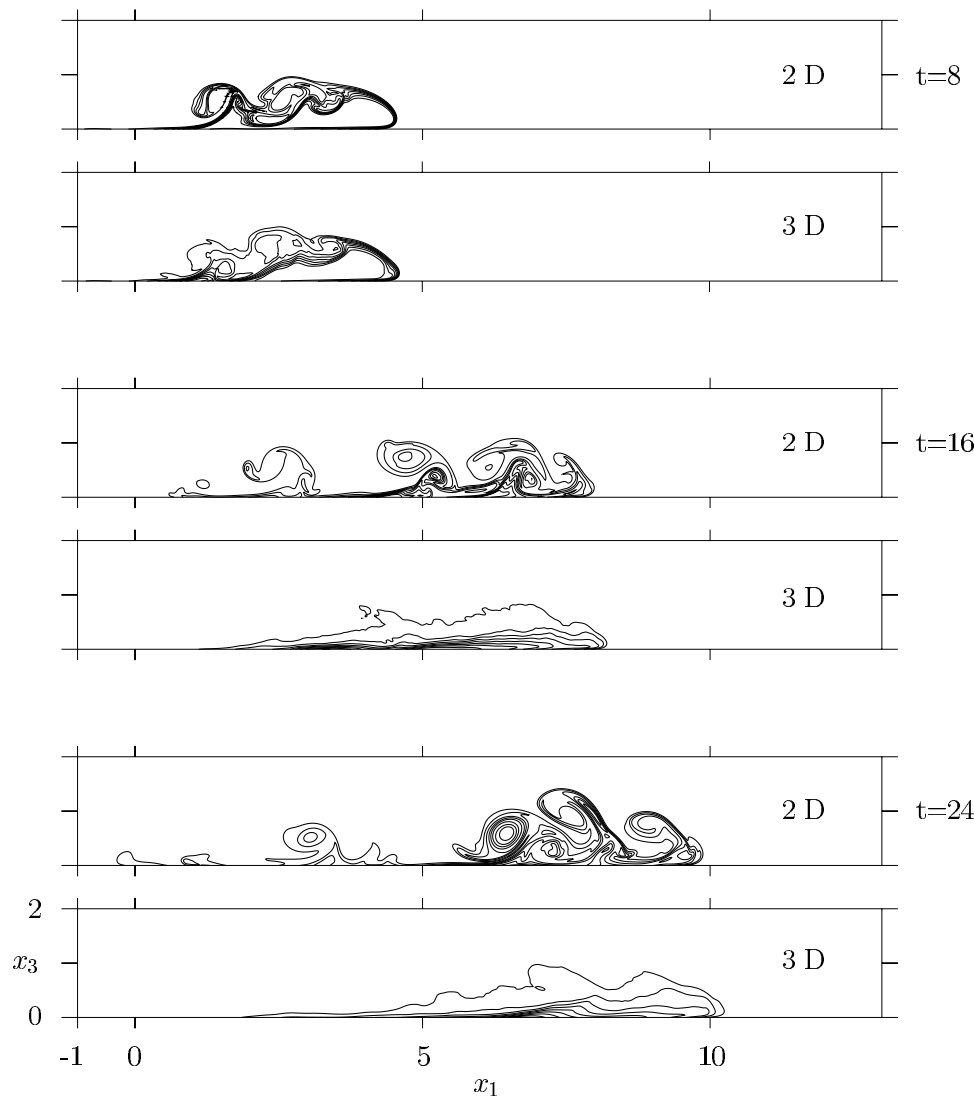

Fig. 10. Isolines of concentration at three different time instants. Comparison of 2D results and spanwise-averaged 3D results (same flow as in Fig. 2). In each plot, the same number of isolines is used with isovalues which are evenly distributed between the maxima and minima of the respective flow fields.

flow, despite the intense turbulence seen in Figs. 2 and 3. This is clearly recognized not only from the results at time $t=8$ in Fig. 10, but also from the other flow quantities we examined. In Figs. 5 and 9 , the respective $2 \mathrm{D}$ results for the front speed, the total mass of suspended particles, and the components of the energy budget are included as dashed lines. In all cases, 2D and 3D results virtually collapse up to $t \approx 10$. This makes it clear that the flow at early times after the release can be accurately predicted by a $2 \mathrm{D}$ model.

In contrast to the early flow stages, more substantial differences between the $2 \mathrm{D}$ and $3 \mathrm{D}$ results appear in the later flow evolution. This is already seen from the isocontours at times 16 and 24 shown in Fig. 10. A striking feature of the 2D flow is the emergence (and persistence) of large and energetic vortices which are completely absent in the 3D case. These vortices can transport particles high up in the vertical direction, which may explain why we found the last few percent of the particles to remain suspended noticeably longer in the $2 \mathrm{D}$ case than in the $3 \mathrm{D}$ case. However, more important is the effect of the large interfacial vortices on the energy budget of the flow. While in the 3D case the development of small-scale turbulence leads to the rapid dissipation of kinetic energy, the $2 \mathrm{D}$ vortical structures are much less affected by viscosity. In consequence, the 
kinetic energy decays much slower in the 2D than in the 3D flow. This is seen in Fig. 9 after about $t=10$. Note that part of the additional kinetic energy available is consumed by lifting up particles which leads to higher levels of potential energy in the $2 \mathrm{D}$ case at late times.

The final sedimentation profiles of the 2D and 3D simulation are compared in Fig. 11 where a good agreement is seen. The curves show that the final runout length of the front is somewhat larger in the 2D than in the 3D case. Given the differences seen in Fig. 5 concerning the front speed for $t \leqslant 60$, this result may seem surprising. However, Fig. 5 also shows that in the $2 \mathrm{D}$ case more particles are still in suspension in the final stage of the flow such that the $2 \mathrm{D}$ front comes to rest later. Most of these remaining particles are localized around the leading part of the front where they eventually settle.

In conclusion, the present results show that the early stage of the flow, which is largely determined by the dynamics of the initial collapse, is well described by a 2D model. Here, 3D effects, although being important locally, have only a minor influence on overall features of the flow. On the other hand, caution is necessary when $2 \mathrm{D}$ simulations are employed to study aspects of the long-time flow evolution, which may develop rather differently in 2D and 3D settings. The reason is that the pronounced vortices that develop in the $2 \mathrm{D}$ case decay much slower than a $3 \mathrm{D}$ turbulence field, maintaining a large-scale fluid motion for long times after all particles have settled.

\section{Resuspension}

If the flow induced is sufficiently vigorous, particle-driven gravity currents may resuspend some of the material that was previously deposited, or even erode an existing sediment bed over which

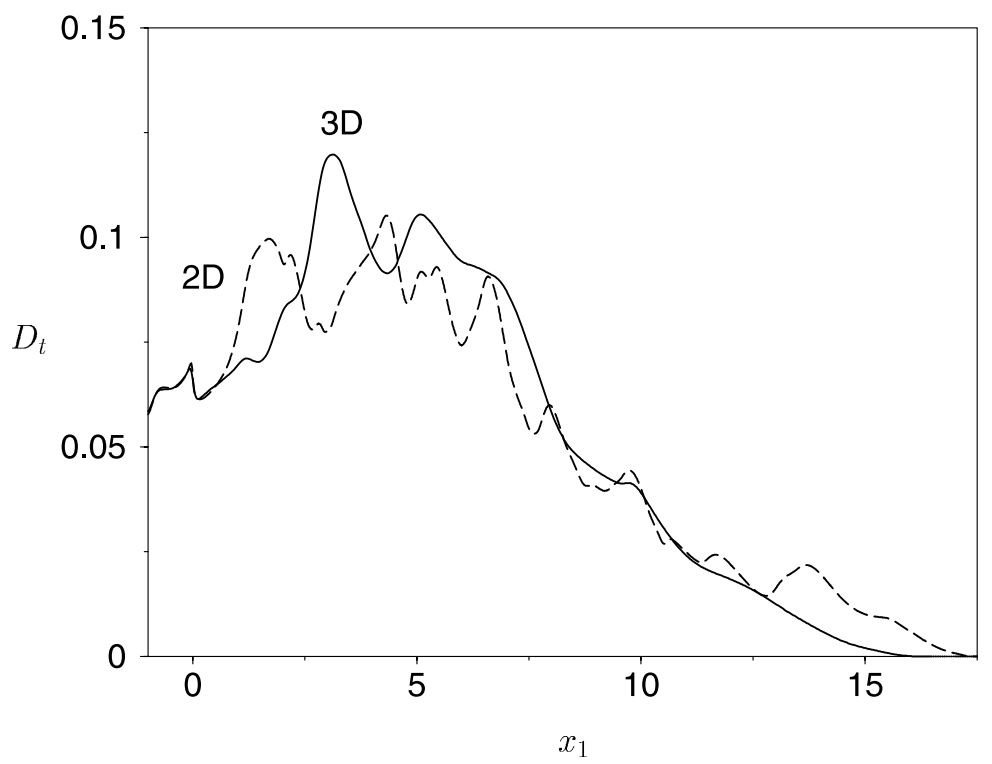

Fig. 11. Non-dimensional deposit $D_{t}$ according to Eq. (21) as a function of $x_{1}$. Final profiles as obtained from a 2D simulation (dashed line) and a 3D simulation (spanwise-averaged results, same flow as in Fig. 2). 
they run. In the present simulations resuspension is not taken into account because, as we will show now, it is not likely to occur in the parameter range studied. Based on the simulation data obtained, we can assess in an a posteriori fashion if and where the flow state exceeds a critical threshold which leads to either incipient motion or resuspension of particles. It should be pointed out that the definition of this critical state is generally a difficult task and a variety of strategies were proposed in the past (for a review, see Ziskind et al., 1995). Here, we will follow the approach of Shields (1936), Mantz (1973), and Yalin and Karahan (1979), in which the critical state is defined by means of a dimensionless wall shear stress $Y_{\mathrm{cr}}$ and a particle Reynolds number $X_{\mathrm{cr}}$

$$
\begin{aligned}
Y_{\mathrm{cr}} & =\frac{\tilde{\rho} \tilde{u}_{\tau}^{2}}{\left(\tilde{\rho}_{\mathrm{p}}-\tilde{\rho}\right) \tilde{g} \tilde{d}_{\mathrm{p}}}, \\
X_{\mathrm{cr}} & =\frac{\tilde{u}_{\tau} \tilde{d}_{\mathrm{p}}}{\tilde{v}} .
\end{aligned}
$$

In the above equations $\tilde{u}_{\tau}$ denotes the friction velocity which is computed from the wall shear stress as

$$
\tilde{u}_{\tau}=\sqrt{\frac{\left|\tilde{\tau}_{w}\right|}{\tilde{\rho}}}, \quad \text { where }\left|\tilde{\tau}_{w}\right|=\left.\tilde{\mu} \sqrt{\left(\frac{\partial \tilde{u}_{1}}{\partial \tilde{x}_{3}}\right)^{2}+\left(\frac{\partial \tilde{u}_{2}}{\partial \tilde{x}_{3}}\right)^{2}}\right|_{\tilde{x}_{3}=0} .
$$

With the characteristic scales introduced in Section 2, the dimensionless friction velocity reads

$$
u_{\tau}=\frac{\tilde{u}_{\tau}}{\tilde{u}_{\mathrm{b}}} .
$$

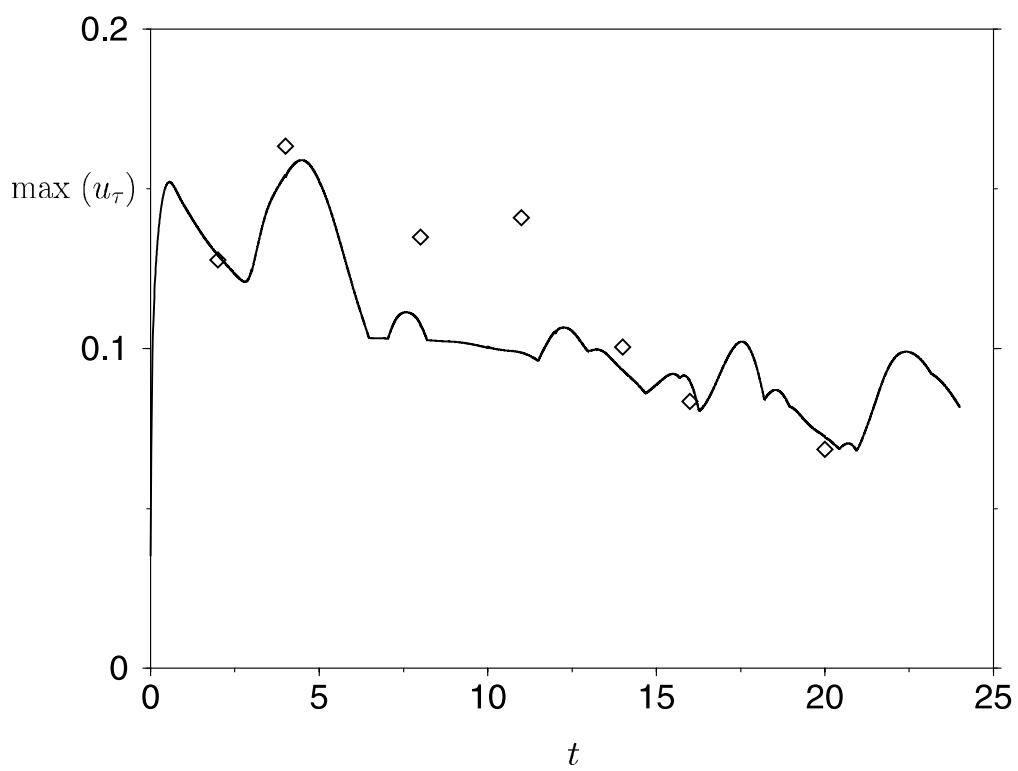

Fig. 12. Temporal development of the maximum (over bottom wall) of the friction velocity. Results for 2D simulation (solid line) and 3D simulation (symbols, same flow as in Fig. 2). 
The time history of the maximum of $u_{\tau}$ over the entire bottom wall is shown in Fig. 12 for the 3D simulation and the corresponding $2 \mathrm{D}$ simulation discussed in the previous section. It is seen that in both cases the largest friction velocities occur at about $t=4$, that is immediately after the reflection of the upper front at the left boundary. In the subsequent flow evolution, $u_{\tau}$ drops by almost a factor of two, which makes it clear that in the present configuration the starting period is the most critical time with respect to possible resuspension. To illustrate where the maximum wall friction occurs, Fig. 13 shows $u_{\tau}$ as a function of the streamwise coordinate at $t=4$ for the 2D flow. It is seen that the maximum is located near the left end of the channel directly underneath the intense vortex. Large shear stresses occur also in the nose region of the front, but the corresponding maximum in $u_{\tau}$ is substantially smaller than the peak value at the rear end at this time. However, the picture changes at later times, when the front is fully developed. Fig. 14 illustrates the spatial distribution of $u_{\tau}$ by a gray-scale coding where darker values indicate larger friction velocities. At the earliest time $(t=4)$ spanwise uniform regions of high wall friction exist that coincide with the maxima already seen in the $2 \mathrm{D}$ results in Fig. 13. Some non-uniformities are only observed at the head of the front, where the 3D motion associated with the lobe-and-cleft instability leads to a streaky pattern. In the subsequent flow development, the high values of $u_{\tau}$ in
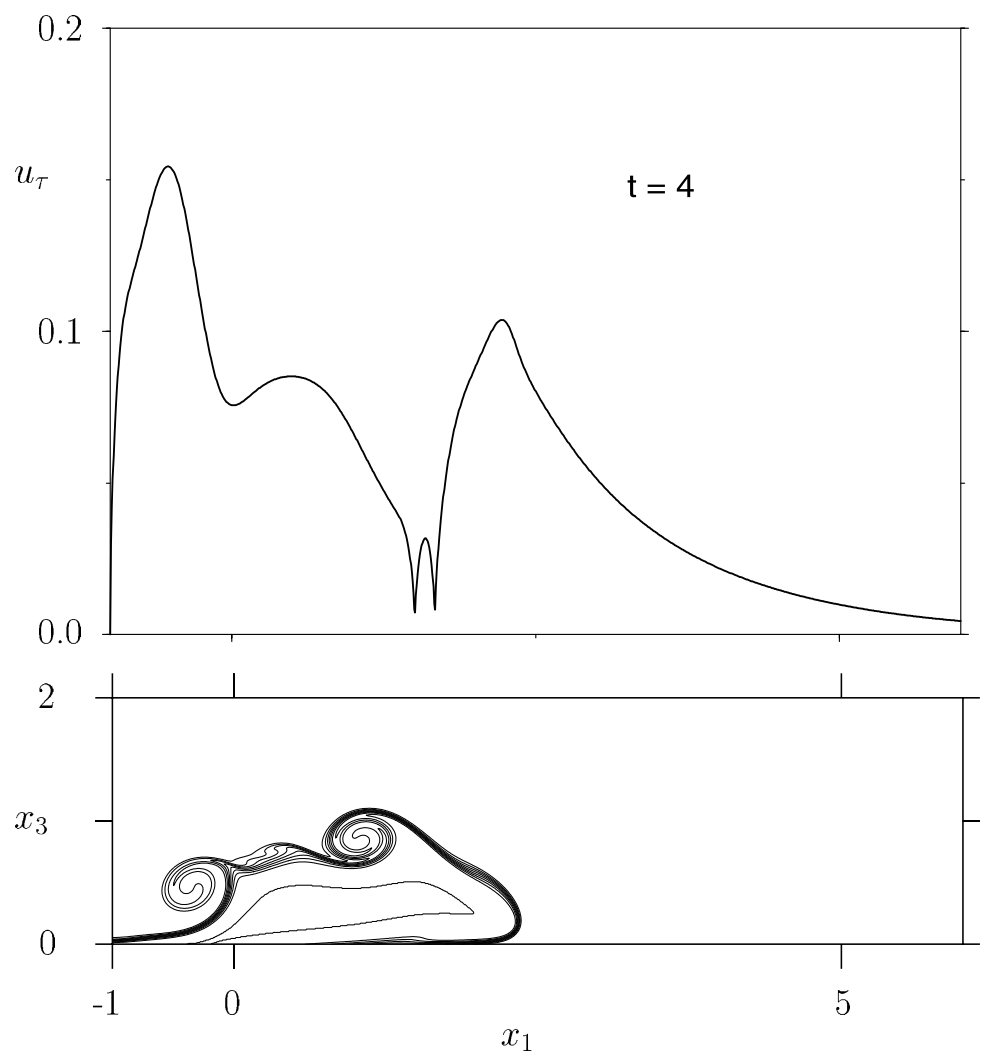

Fig. 13. Top: Friction velocity $u_{\tau}$ at time $t=4$ as a function of streamwise coordinate $x_{1}$. Bottom: Isolines of concentration for the same time instant. Results obtained from a 2D simulation at $G r=5 \times 10^{6}$. 

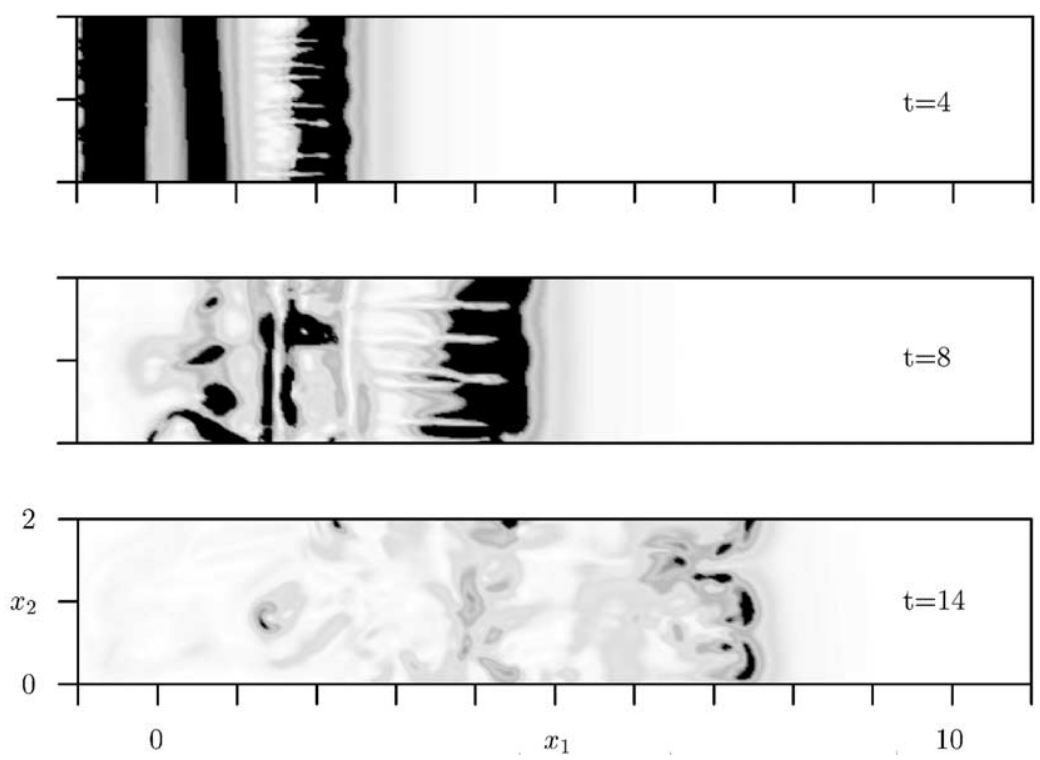

Fig. 14. Structure of the local friction velocity $u_{\tau}$ at the bottom wall visualized by a gray-scale coding. White: $u_{\tau}=0$, black: $u_{\tau}>0.08$. Results for three different times (same flow as Fig. 2).

the rear part quickly diminish, and at $t=14$ the leading edge of the front is the region which experiences the strongest frictional stresses.

The variation of the overall maximum of $u_{\tau}$ with increasing Grashof number is depicted in Fig. 15 as a dashed line. For all Grashof numbers the same non-dimensional settling velocity of 0.02

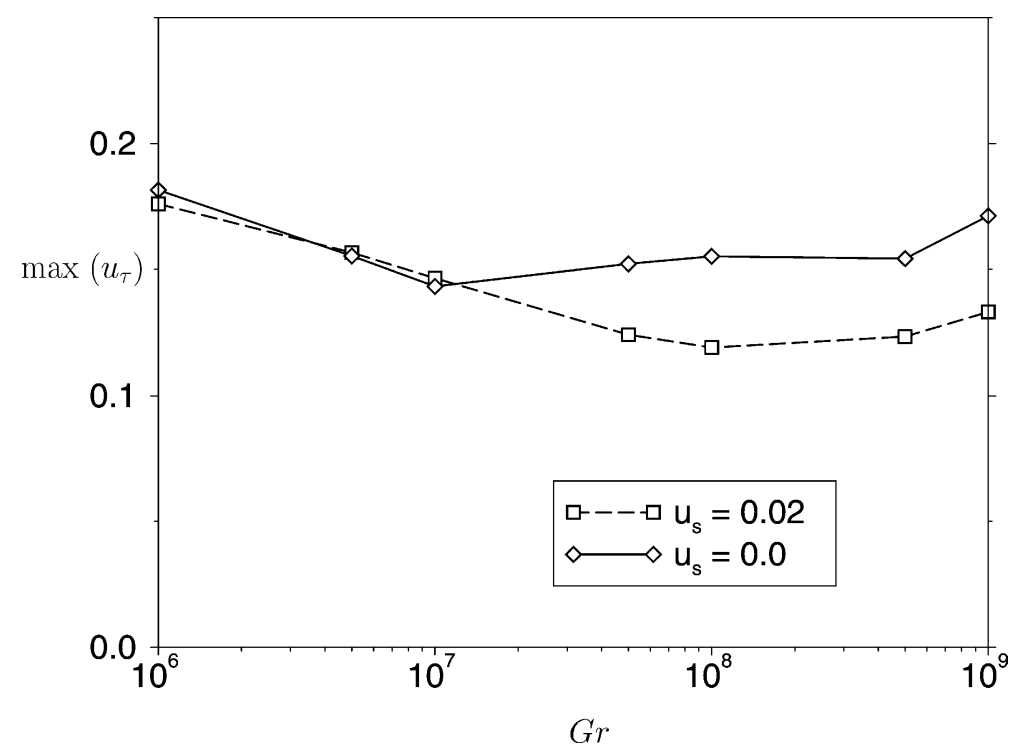

Fig. 15. Overall maximum of friction velocity $u_{\tau}$ at bottom wall. Results for different Grashof numbers. Solid line $u_{\mathrm{s}}=0.0$, dashed line $u_{\mathrm{s}}=0.02$. 
was used, and all results were obtained from 2D simulations. Since the largest wall frictions occur during the first few time units, the use of a $2 \mathrm{D}$ model is justified as long as only the maximum of $u_{\tau}$ is of interest. For comparison, the result for density-driven fronts $\left(u_{\mathrm{s}}=0\right)$ is also included, and it is seen that no large differences exist between the two cases. Both curves show only a modest variation of the maximum of $u_{\tau}$ with Grashof number between $G r=10^{6}$ and $10^{9}$, which corresponds to the range of Grashof numbers encountered in typical laboratory experiments. To a good approximation, the maximum in friction velocity can be taken as constant in this regime with a value of around 0.15. Based on this estimate, we can now assess the issue of resuspension for the conditions in the experiments of de Rooij and Dalziel (1998) that we have used for comparison in Section 4. In these experiments silicon carbide particles with a density of $\tilde{\rho}_{\mathrm{p}}=$ $3.217 \mathrm{~g} / \mathrm{cm}^{3}$ and a diameter of $\widetilde{d}_{\mathrm{p}}=37 \mu \mathrm{m}$ were used. $50 \mathrm{~g}$ of these particles were suspended in a reservoir of $26.5 \times 26 \times 10 \mathrm{~cm}^{3}$ filled with tap water, which results in a Grashof number of $G r=10^{8}$ and a buoyancy velocity of $\tilde{u}_{\mathrm{b}}=0.08 \mathrm{~m} / \mathrm{s}$. From Eq. (33) a maximum frictional velocity of $\tilde{u}_{\tau}=0.012 \mathrm{~m} / \mathrm{s}$ is computed using the average value of $u_{\tau}=0.15$. As dimensionless shear stress $Y_{\mathrm{cr}}$ we then obtain $Y_{\mathrm{cr}}=0.18$, and for the particle Reynolds number we find $X_{\mathrm{cr}}=0.44$. According to Mantz (1973), the limit for the onset of resuspension is $Y_{\mathrm{cr}} \approx 0.4$ at this particle Reynolds number which is well above the value obtained for the conditions in the experiment. On the other hand, incipient motion of particles, for which Mantz gives a limit of $Y_{\mathrm{cr}} \approx 0.07$, may have occurred, though only during the very first stage of the flow, since both $X_{\mathrm{cr}}$ and $Y_{\mathrm{cr}}$ drop substantially when the flow is developed.

\section{Conclusions}

We have presented a computational study of 2D and 3D particle-driven gravity currents in the lock-exchange configuration. The simulations were performed to gain more insight into the flow dynamics than could be obtained from previous experiments. In the present study dilute flows with small particle loadings were considered, and the treatment of the particulate phase was accomplished by a Eulerian approach using a transport equation for the local particle-number density. For the numerical integration of the governing equations a high-order mixed spectral/ spectral-element scheme was employed.

The 3D simulation was performed for a Grashof number of $5 \times 10^{6}$ and it was demonstrated that the flow within the front attains a turbulent state. In the earlier stages of the flow, when a developed head of the front was still discernible, the characteristic lobe-and-cleft structure could be observed at the leading edge. However, after a significant portion of all particles had sedimented, the outline of the front became less and less well-defined. To assess the potential of 2D simulations for the study of particle-driven fronts, we have directly compared results from the 3D simulation with 2D data for the same Grashof number. It was found that during the initial stages the overall features of the flow were accurately captured by the $2 \mathrm{D}$ model. However, concerning the flow structure at later times, more significant differences appeared between 2D and 3D simulations, which suggests that care must be taken if $2 \mathrm{D}$ simulations are employed to study features that depend primarily on the long-time evolution of the flow.

We have investigated the temporal evolution of particle sedimentation at the bottom wall and found the results to be in good agreement with previously published experimental data. For the 
case studied, the integrated mass flux of sedimenting particles increases proportionally to $t^{0.5}$ during the early flow stages. At later times, after about half the particles have settled out, the mass flux rapidly decreases with time, decaying faster than $1 / t^{2}$. The sedimentation of particles is associated with additional dissipative losses in the flow that do not exist in density-driven gravity currents. We have shown that in the present case about $40 \%$ of the initial potential energy in the system is 'lost' due to particle settling, i.e. is not available for convective transport and mixing.

Finally, we have addressed the issue of a possible resuspension of particles. To determine whether or not resuspension can occur, we have adopted a simple approach which employs a suitable particle Reynolds number and the wall-friction velocity as characteristic quantities. It was shown that the maximum friction velocity in the flow occurs during the initial stage before the front is fully developed. An interesting finding is that the ratio between this maximum friction velocity and the buoyancy velocity is roughly constant over the range of Grashof numbers accessible to typical laboratory experiments. Concerning the experiments that we have compared with in the present study, we conclude that resuspension has probably not occurred.

\section{Acknowledgements}

Most of the present simulations have been performed on the NEC SX-5 system of the Swiss Center for Scientific Computing (SCSC) in Manno, TI. The authors wish to thank the SCSC staff for support with the optimization of the simulation code. Thanks are also due to J. Moser for his help with the resuspension study.

\section{References}

Bonnecaze, R.T., Huppert, H.E., Lister, J.R., 1993. Particle-driven gravity currents. J. Fluid Mech. 250, $339-369$.

Britter, R.E., Simpson, J.E., 1978. Experiments on the dynamics of a gravity current head. J. Fluid Mech. 88, $223-240$.

Dade, W.B., Huppert, H.E., 1994. Predicting the geometry of channelised deep-sea turbidites. Geology $22,645-648$.

Dade, W.B., Huppert, H.E., 1995. A box model for non-entraining, suspension-driven gravity surges on horizontal surfaces. Sedimentology 42, 453-471.

Davis, R.H., Hassen, M.A., 1988. Spreading of the interface at the top of a slightly polydisperse sedimenting suspension. J. Fluid Mech. 196, 107-134.

de Rooij, F., Dalziel, S.B., 1998. Time-resolved measurements of the deposition under turbidity currents. In: Proceedings of the Conference on Sediment Transport and Deposition by Particulate Gravity Currents, Leeds, September 7-9 .

Gladstone, C., Phillips, J.C., Sparks, R.S.J., 1998. Experiments on bidisperse, constant-volume gravity currents: propagation and sediment deposition. Sedimentology 45, 833-843.

Ham, J.M., Homsy, G.M., 1988. Hindered settling and hydrodynamic dispersion in quiescent sedimenting suspensions. Int. J. Multiphase Flow 14, 533-546.

Härtel, C., Kleiser, L., Michaud, M., Stein, C.F., 1997. A direct numerical simulation approach to the study of intrusion fronts. J. Eng. Math. 32, 103-120.

Härtel, C., Meiburg, E., Necker, F., 1999. Vorticity dynamics during the start-up phase of gravity currents. Il Nuovo Cimento C 22, 823-833.

Härtel, C., Meiburg, E., Necker, F., 2000a. Analysis and direct numerical simulation of the flow at a gravity-current head. Part 1: Flow topology and front speed for slip and no-slip boundaries. J. Fluid Mech. 418, $189-212$.

Härtel, C., Carlsson, F., Thunblom, M., 2000b. Analysis and direct numerical simulation of the flow at a gravitycurrent head. Part 2: The lobe-and-cleft instability. J. Fluid Mech. 418, 213-229. 
Hallworth, M.A., Hogg, A.J., Huppert, H.E., 1998. Effects of external flow on compositional and particle gravity currents. J. Fluid Mech. 359, 109-142.

Hogg, A.J., Ungarish, M., Huppert, H.E., 2000. Particle-driven gravity currents: asymptotic and box model solutions. Eur. J. Mech. B 19, 139-165.

Huppert, H.E., Simpson, J.E., 1980. The slumping of gravity currents. J. Fluid Mech. 99, 785-799.

Huppert, H.E., 1986. The intrusion of fluid mechanics into geology. J. Fluid Mech. 173, 557-594.

Issler, D., Gauer, P., Barbolini, M., 1998. Continuum models of particle entrainment and deposition in snow drift and avalanche dynamics. In: Proceedings of the Conference on Models of Continuum Mechanics in Analysis and Engineering, Darmstadt, October.

Lázaro, B.J., Lasheras, J.C., 1989. Particle dispersion in a turbulent, plane, free shear layer. Phys. Fluids A 1, 10351044.

Mantz, P.A., 1973. Cohesionless, fine graded, flaked sediment transport by water. Nature Phys. Sci. 246, 14-16.

Maxey, M.R., Riley, J.J., 1983. Equations of motion for a small rigid sphere in a nonuniform flow. Phys. Fluids 26, 883-889.

Middleton, G.V., 1993. Sediment deposition from turbidity currents. Annu. Rev. Earth Planet. Sci. 21, 89-114.

Parker, G., Fukushima, Y., Pantin, H.M., 1986. Self-accelerating turbidity currents. J. Fluid Mech. 171, 145-181.

Rottman, J.W., Simpson, J.E., 1983. Gravity currents produced by instantaneous releases of a heavy fluid in a rectangular channel. J. Fluid Mech. 135, 95-110.

Shields, A., 1936. Anwendung der Ähnlichkeitsmechanik und der Turbulenzforschung auf die Geschiebebewegung, Mitteilungen der (Preussischen) Versuchsanstalt für Wasserbau und Schiffbau, vol. 26, Berlin.

Simpson, J.E., 1972. Effects of the lower boundary on the head of a gravity current. J. Fluid Mech. 53, 759-768.

Simpson, J.E., 1997. Gravity Currents in the Environment and the Laboratory, second ed. Cambridge University Press, Cambridge.

Winters, K.B., Lombard, P.N., Riley, J.J., D'asaro, E.A., 1995. Available potential energy and mixing in densitystratified fluids. J. Fluid Mech. 289, 115-128.

Yalin, M.S., Karahan, E., 1979. Inception of sediment transport. J. Hydraulics Division (Proc. ASCE) 105 (HY11), $1433-1443$.

Ziskind, G., Fichman, M., Gutfinger, C., 1995. Resuspension of particulates from surfaces to turbulent flows - review and analysis. J. Aerosol. Sci. 26, 613-644. 